\title{
THE ECOLGITE CONDUNDRUM AND \\ THE THERMAL EVOLUTION OF MOUNTAIN BELTS
}

Mike Bickle, University Cambridge

1) Thermal modelling used to be simple - there was plenty of time.

"A preliminary thermal model for regional metamorphism in the Eastern Alps, 1975, EPSL"

2) Metamorphic pressures of crustal rocks have increased! How are they exhumed?

3) Synchronous emplacement of high/ultra-high pressure rocks questions models for thermal evolution of mountain belts. 


\section{European Alps}

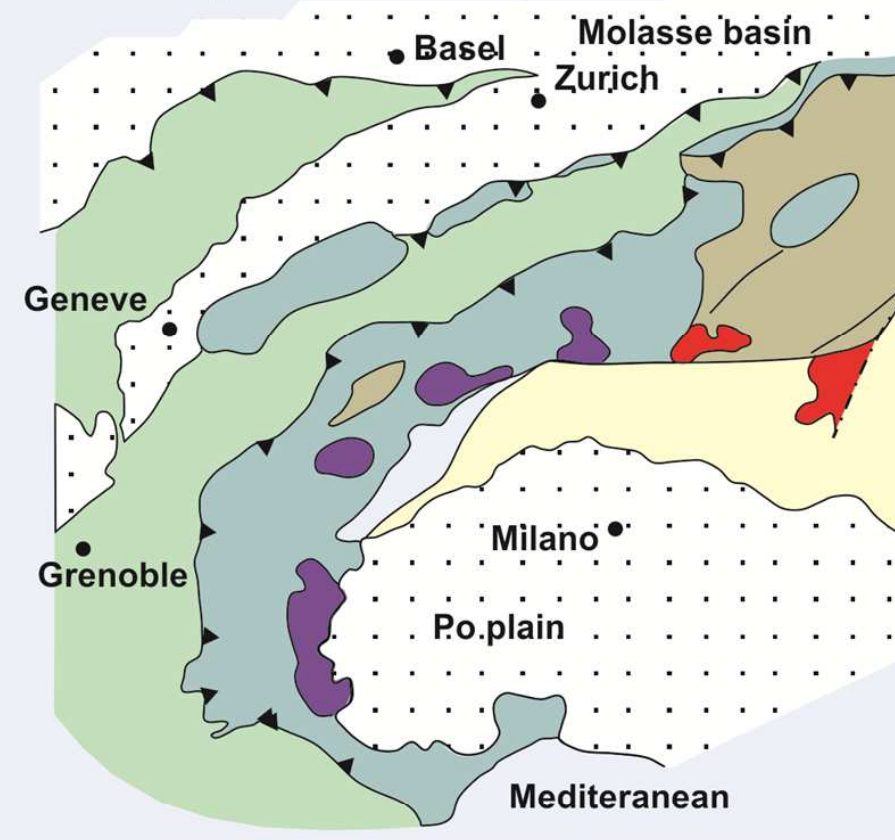

Penninic units

Ultra-high pressure

Helvetic units

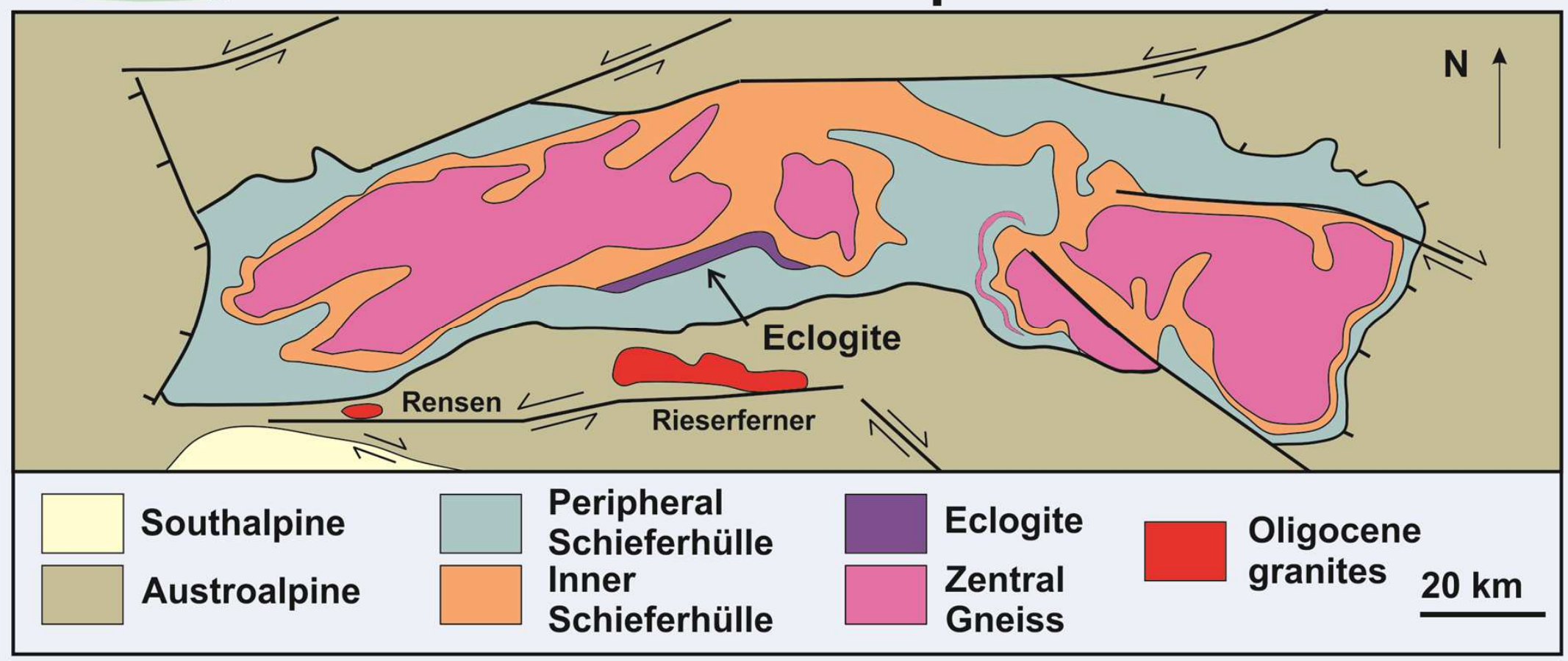

Austroalpine

Southernalpine

Oligocene granites

Molasse basin

\section{Eastern Alps}




\section{Ages Greenschist-Amphibolite facies Barrovian metamorphism}

$\mathrm{U}-\mathrm{Pb}$ apatite

(450 ${ }^{\circ} \mathrm{C}$ )

Sneider et al. 2015
$\mathrm{Rb}-\mathrm{Sr}$ white mica $\left(550^{\circ} \mathrm{C}\right)$

- Reddy et al. 1993, Glodny et al., 2005, Gleisner et al., (2007) 27-31, $29.8 \pm 0.5,31.5 \pm 0.5$.
$\mathrm{Th}-\mathrm{Pb}$ allanite

$27.7 \pm 0.7$ Cliff et al. 2015: $D_{A}^{2}$
Sm-Nd garnet $25.7 \pm 0.9$ isochron. Favaro et al., 2015 post-dating $D_{A}^{2}$
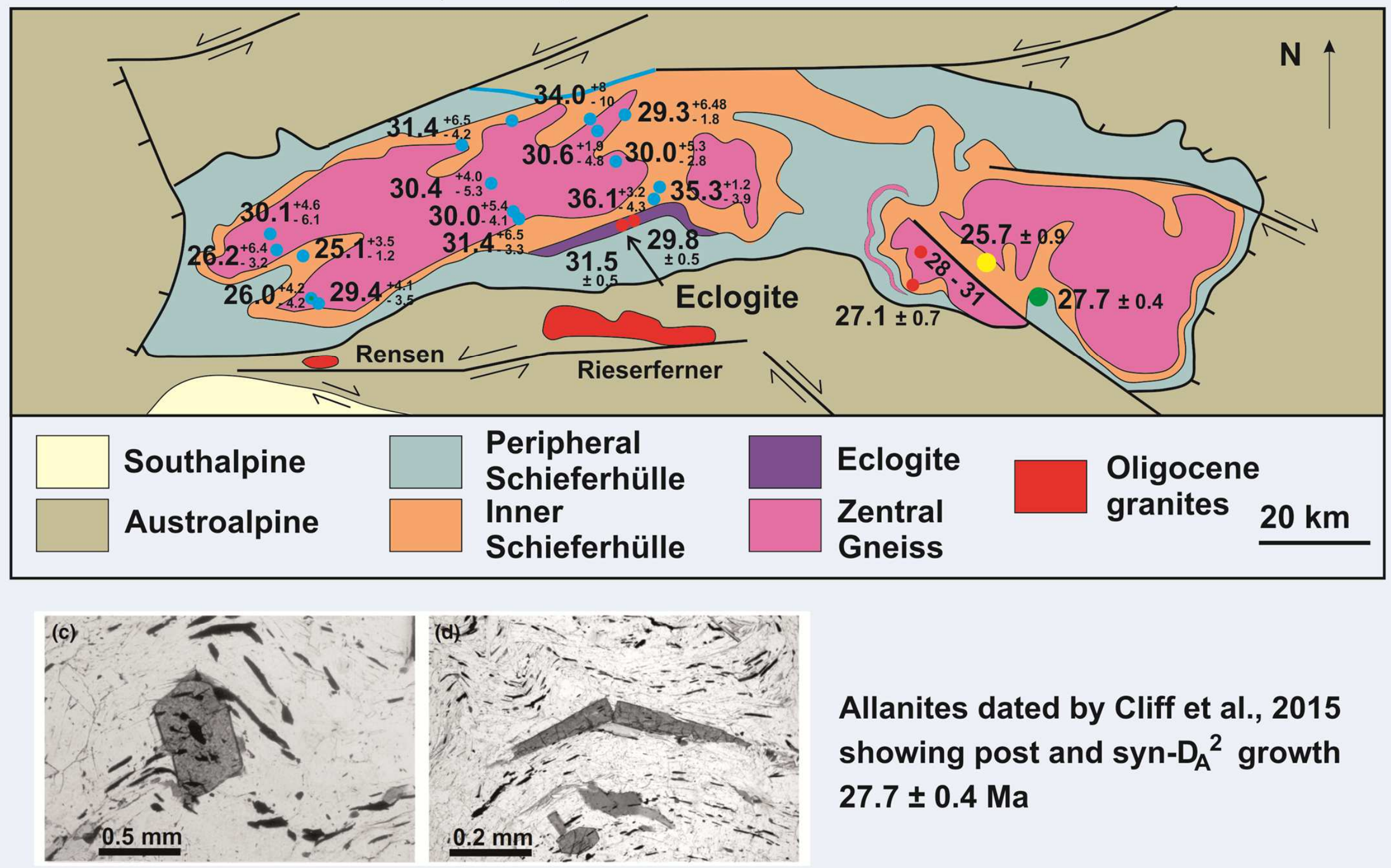

Allanites dated by Cliff et al., 2015 showing post and syn- $\mathrm{D}_{\mathrm{A}}{ }^{2}$ growth $27.7 \pm 0.4 \mathrm{Ma}$ 


\section{P-T-t paths Tauern Barrovian metamorphism}

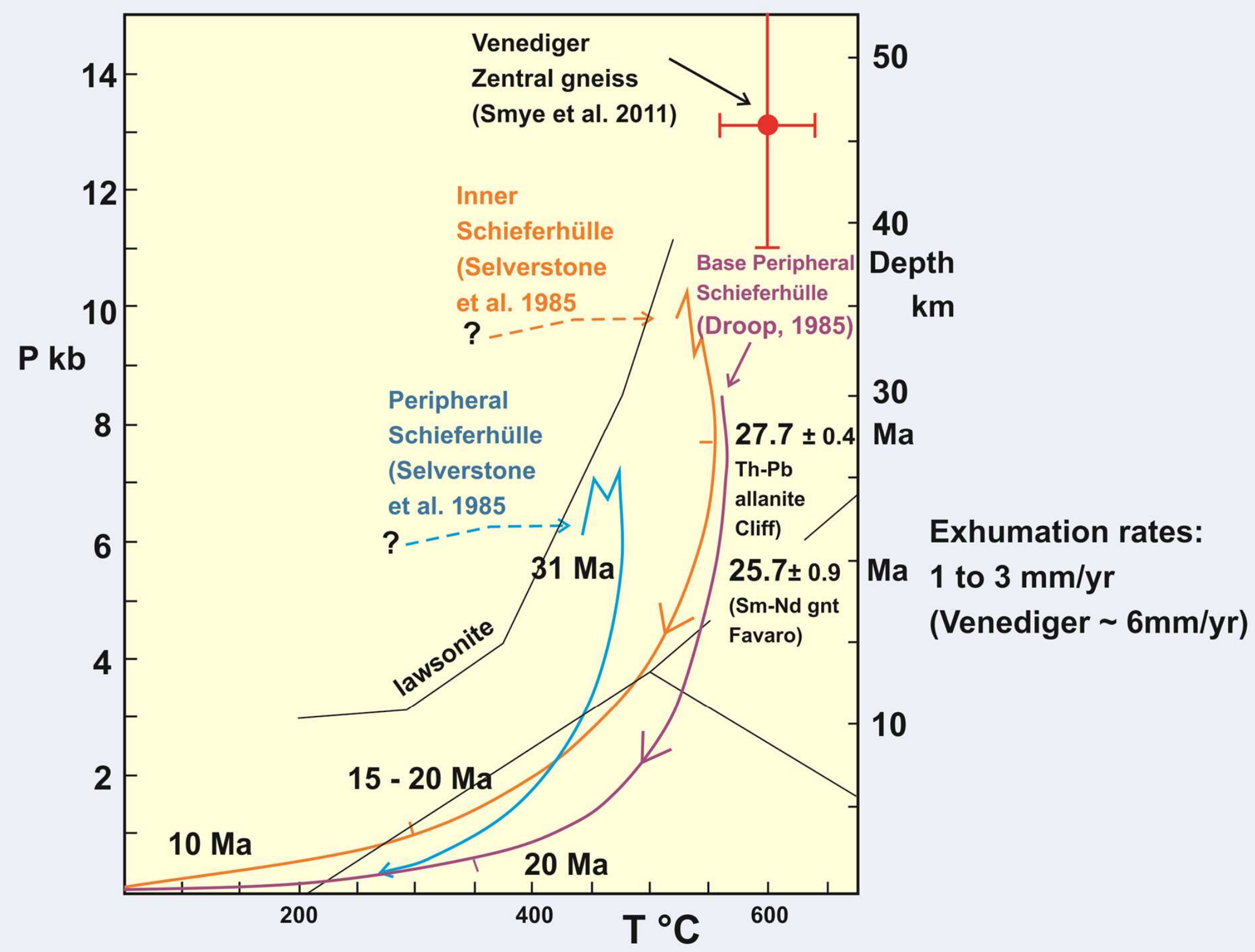




\section{P-T-t paths Ecligite to Barrovian}

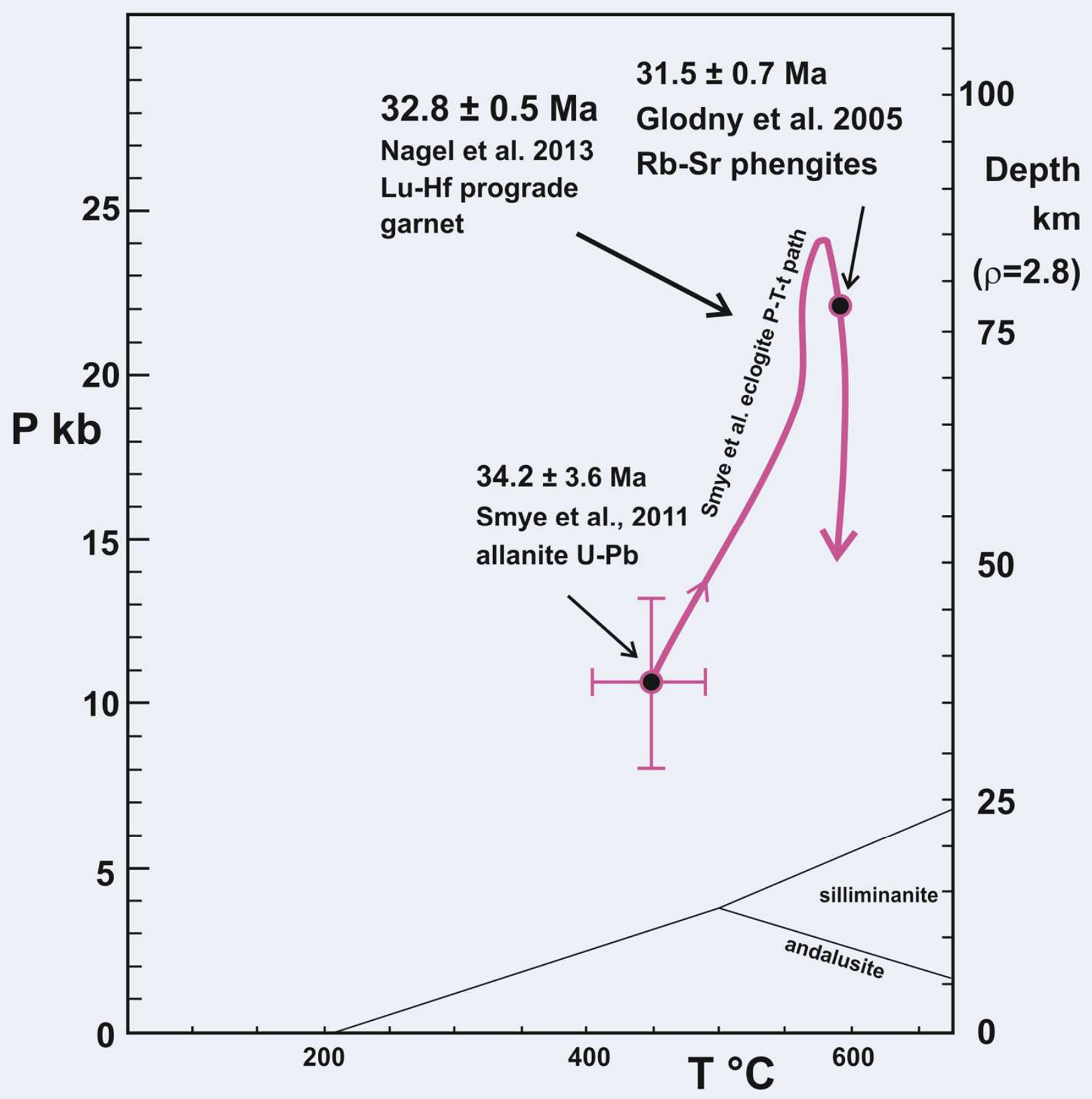




\section{P-T-t paths Ecligite to Barrovian}

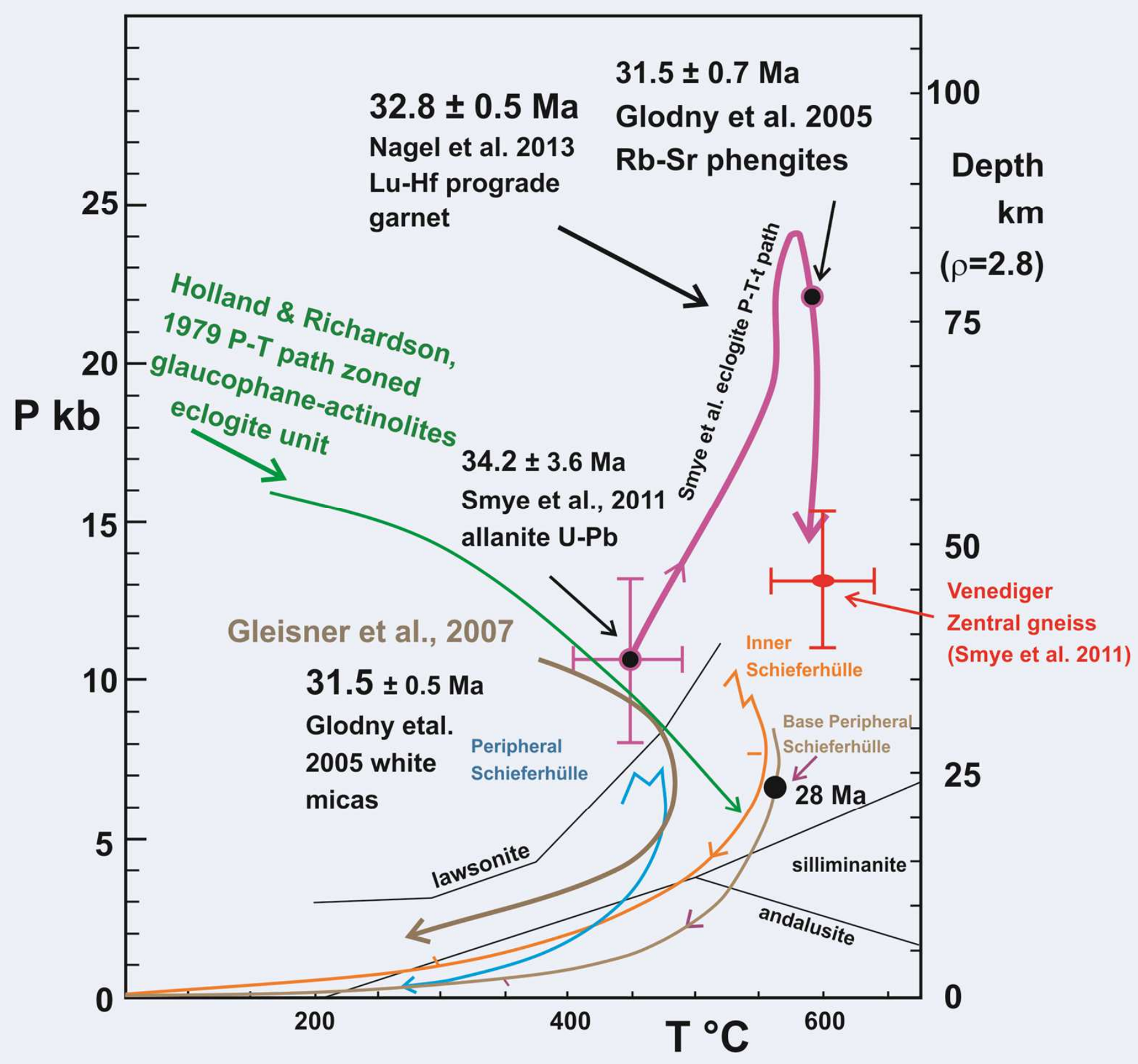




\section{P-T-t paths Ecligite to Barrovian}

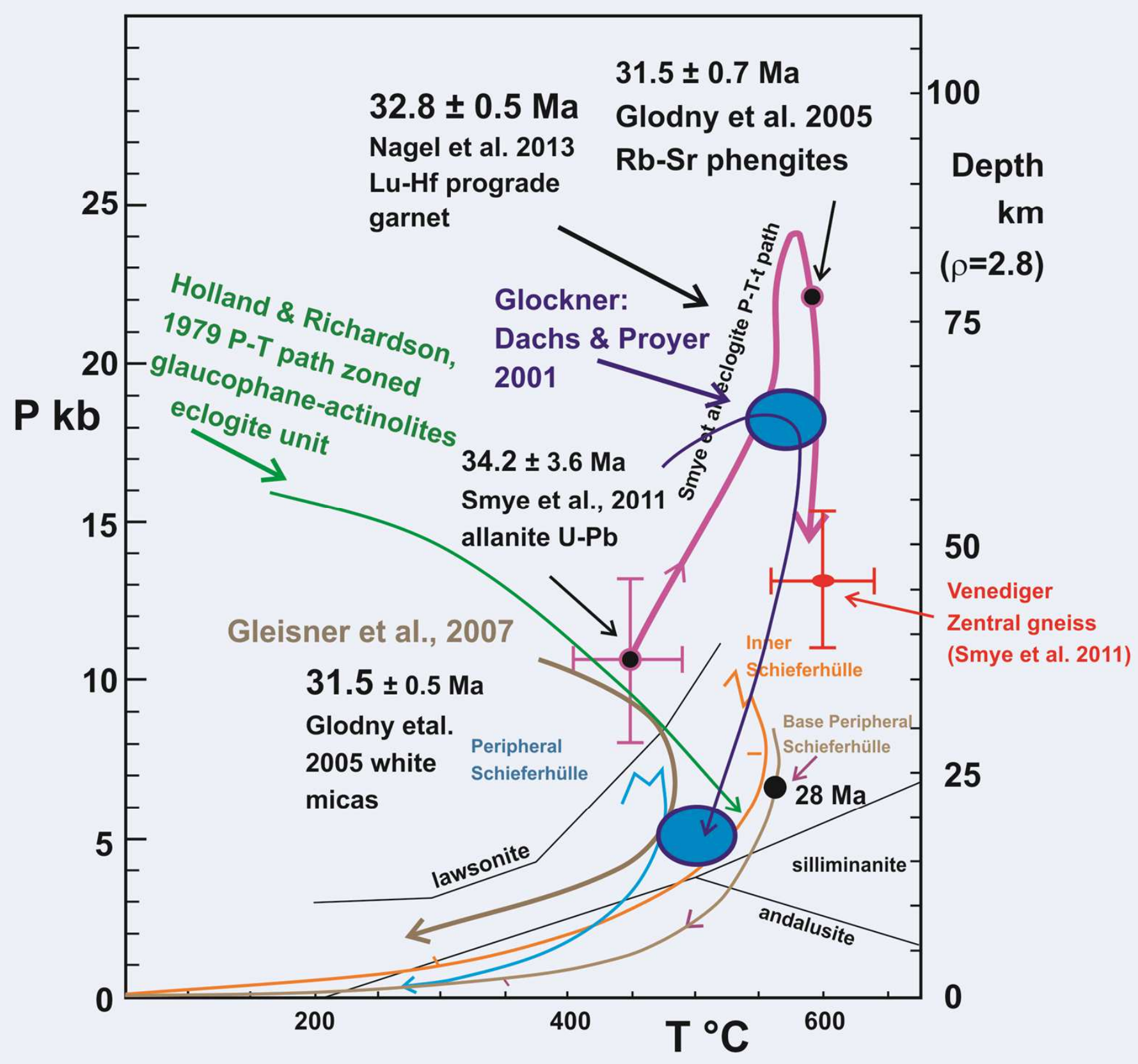




\section{P-T-t paths Ecligite to Barrovian}

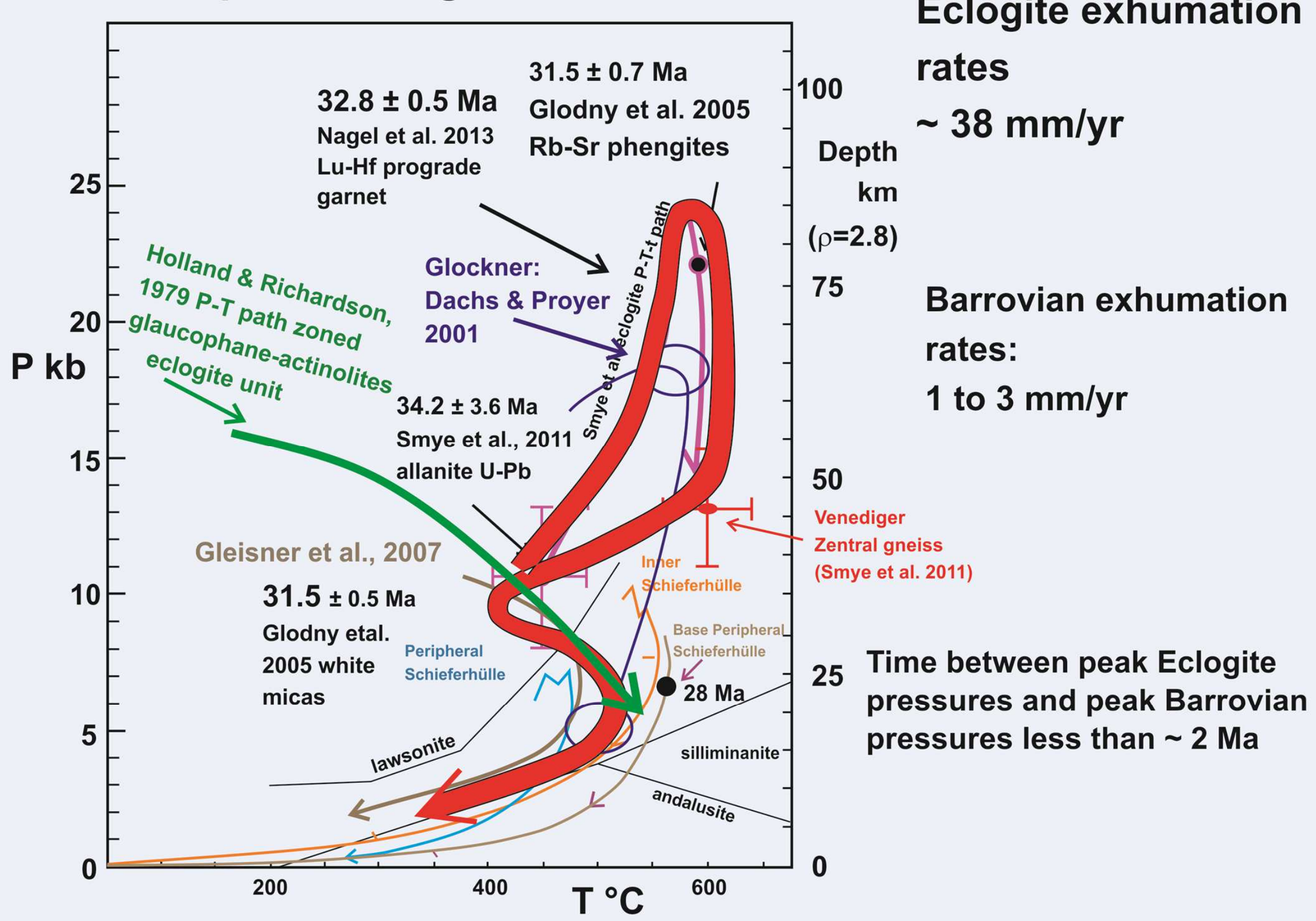

\section{Eclogite exhumation}

rates

Glodny et al. 2005

Depth $\sim 38 \mathrm{~mm} / \mathrm{yr}$

km

Barrovian exhumation

rates:

1 to $3 \mathrm{~mm} / \mathrm{yr}$

50

Zentral gneiss

Smye et al. 2011)

25 Time between peak Eclogite pressures and peak Barrovian pressures less than $\sim 2 \mathrm{Ma}$ 
Thermal model - 30 Ma before exhumation

e.g. Bickle et al. 1975

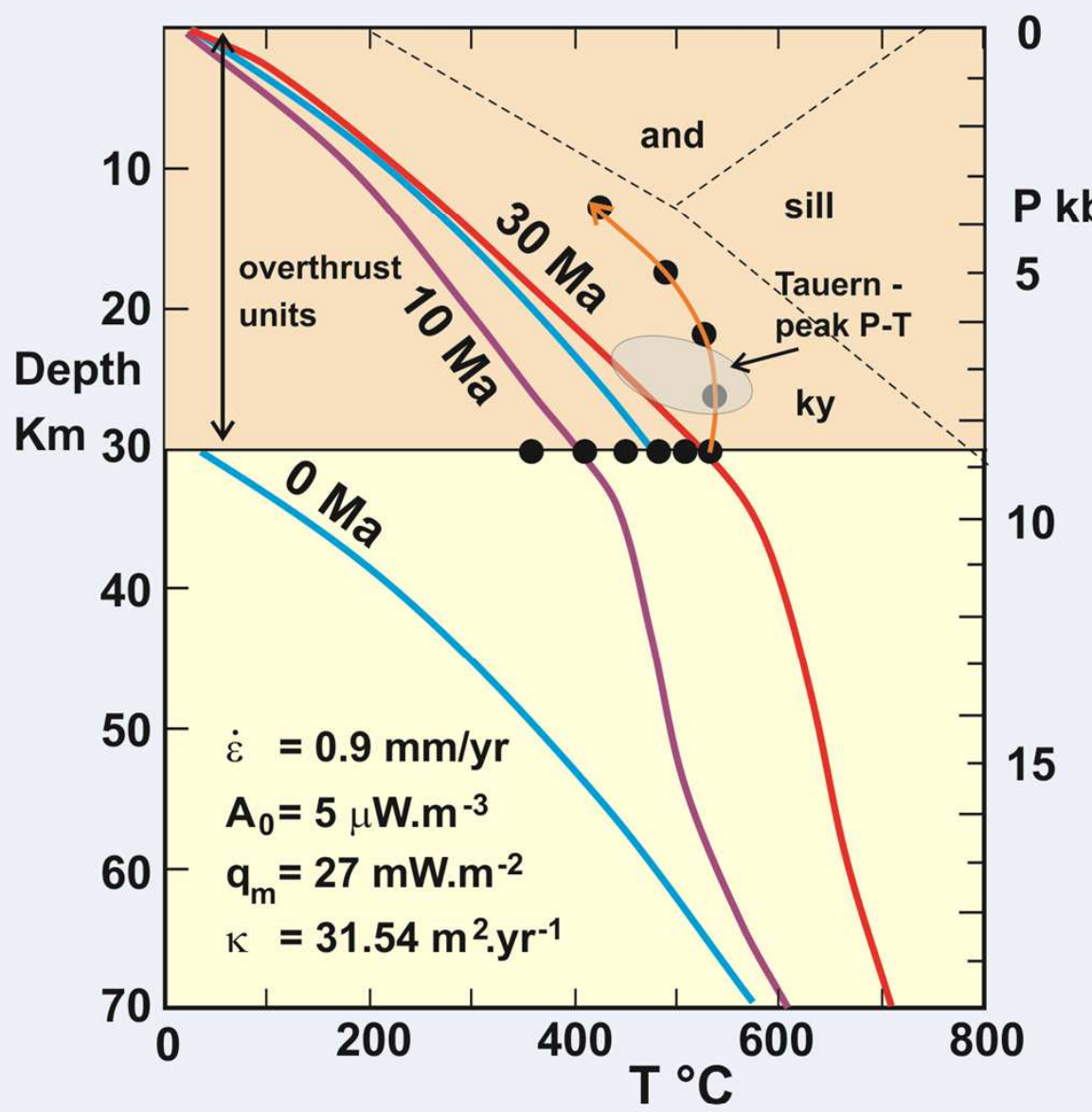

Thermal model - 5 Ma before exhumation at $0.9 \mathrm{~mm} / \mathrm{yr}$ (Smye et al., 2011)

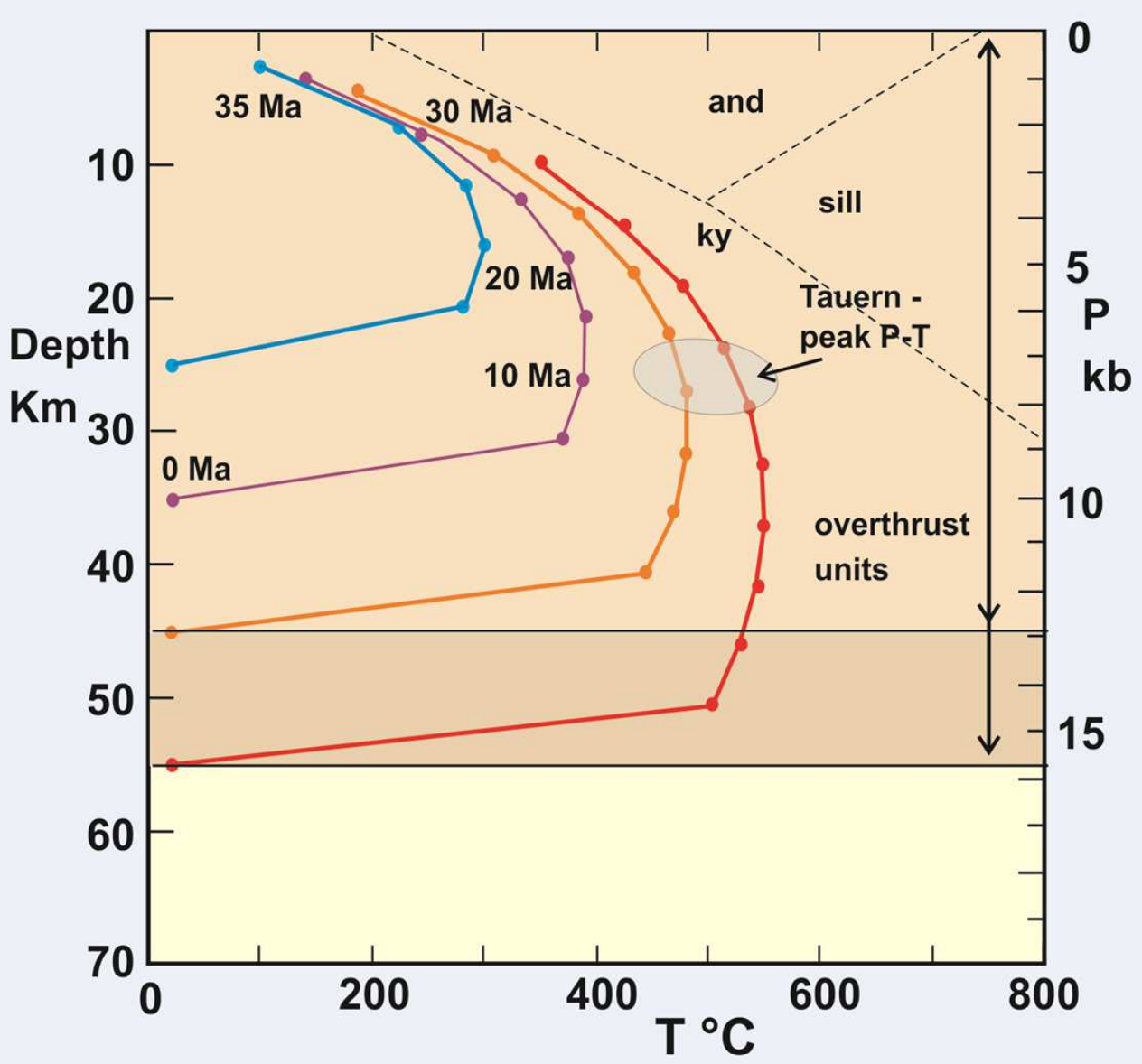

Thicker overburden - faster heating - to a point - needs faster and earlier exhumation

Note: Thick overburden implies crustal thickening 10 Ma before peak 28 Ma conditions and much exhumation faster than $1 \mathrm{~mm} / \mathrm{yr}$ 


\section{Solutions:}

1) Additional heat sources - cools too slowly

2) Overthrust sheet with basal temperature $\sim 600{ }^{\circ} \mathrm{C}$

3) Insert eclogites into thickened crust.

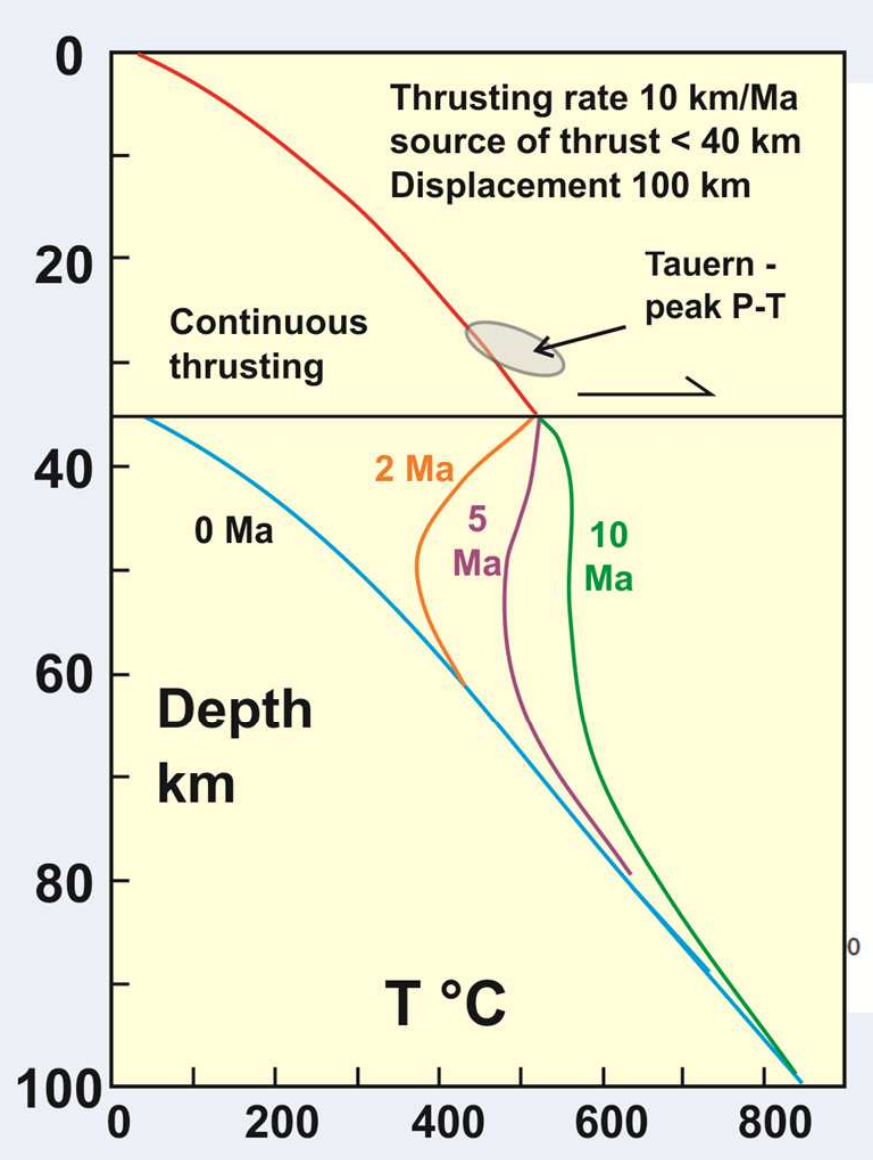

Continuous thrusting -

1) Requires base of thrust not far from hot source.

2) Needs thrust sheet rapidly exhumed huge amount of sediment produced.

3) Evidence that base of Altkristallin sheet cooled below $350{ }^{\circ} \mathrm{C}$ (biotite $\mathrm{Rb}-\mathrm{Sr}$ ages at $65 \mathrm{Ma}$, Brewer, 1969).

4) Peak temperatures at base of Altkristallin $\sim 420$ C (Bickle et al., 1975). 
From Schlunegger \& Kissling (2015) Nature Communications - modified from Fry et al, (2010) EPSL

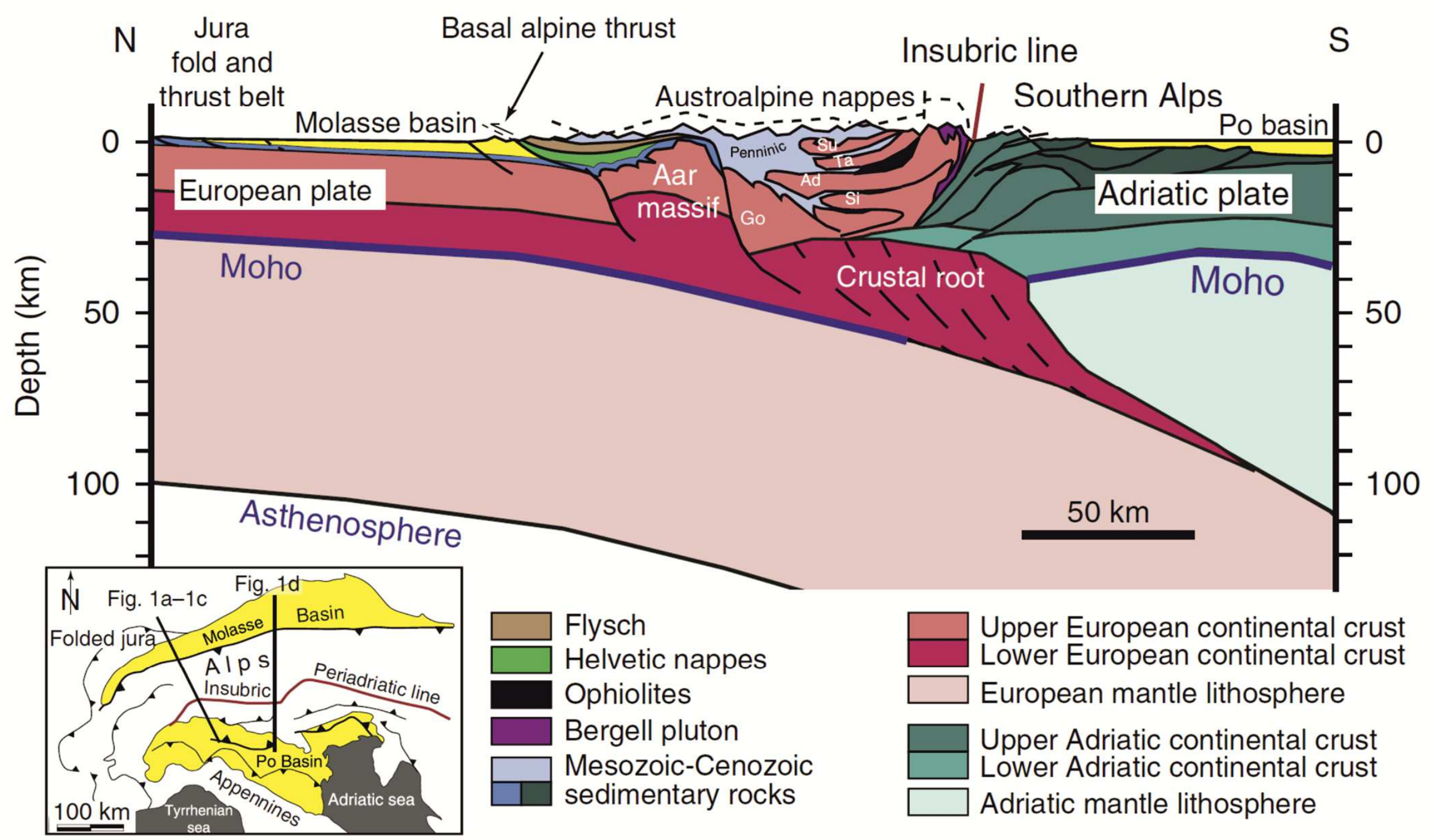




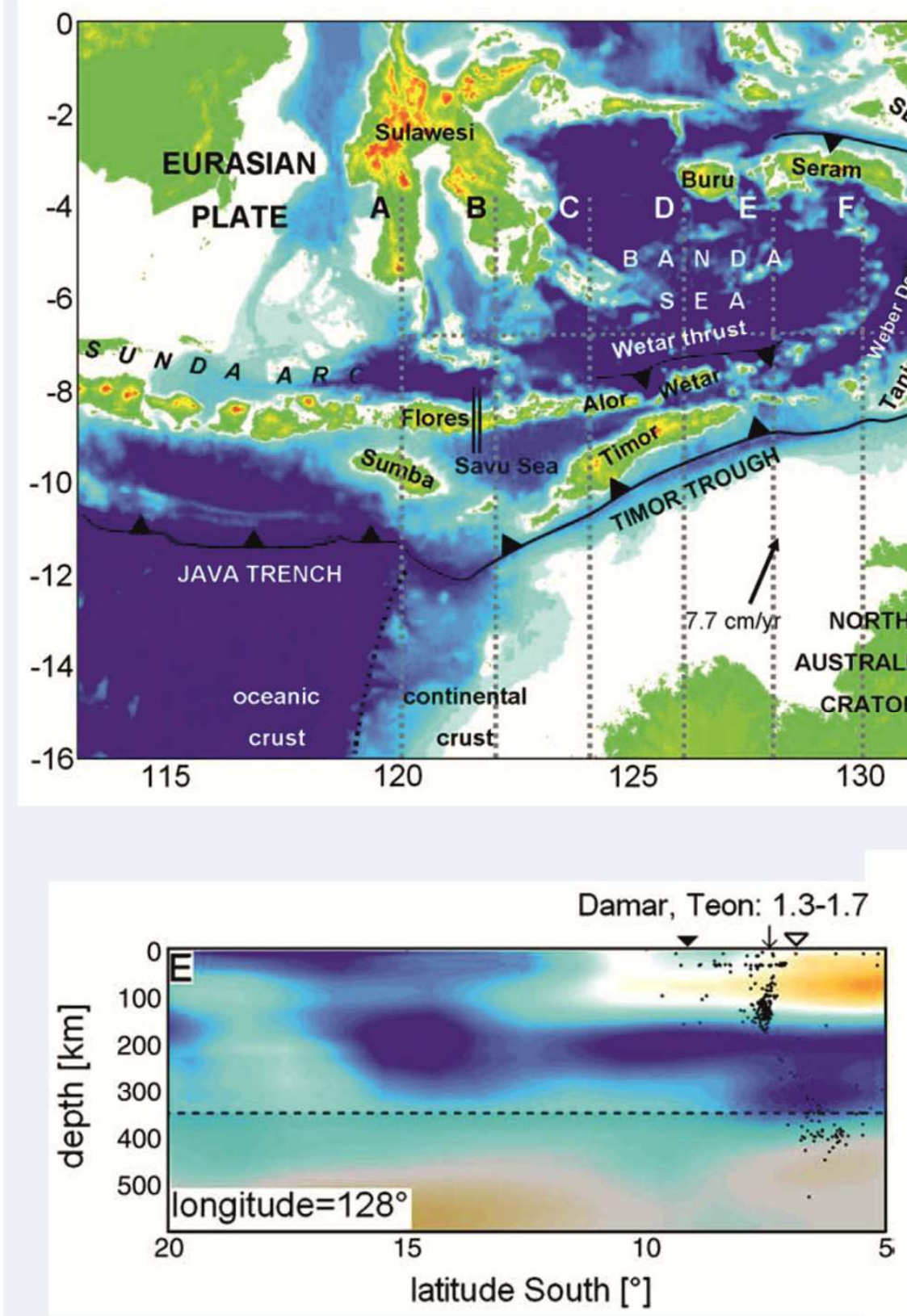

latitude South $\left[^{\circ}\right]$

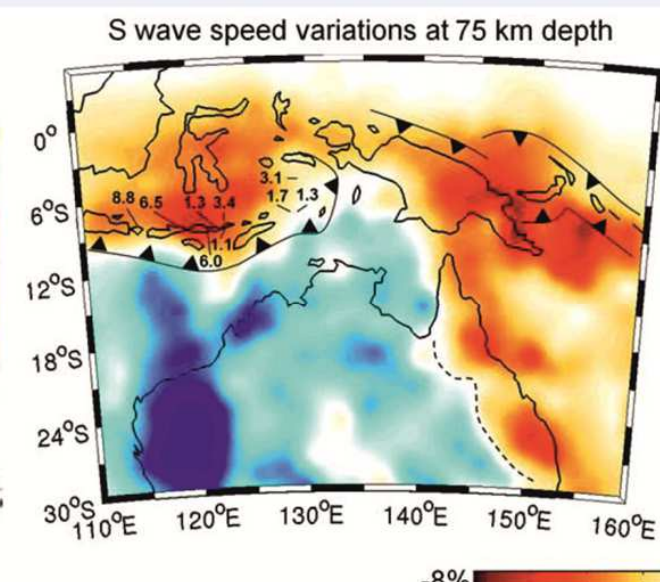

$-8 \%$

\section{Subduction of} continental lithosphere to $\sim 200 \mathrm{~km}$ in Banda Arc. (Fichtner etal. 2010, EPSL).

Tomography (velocities) from full waveform tomography

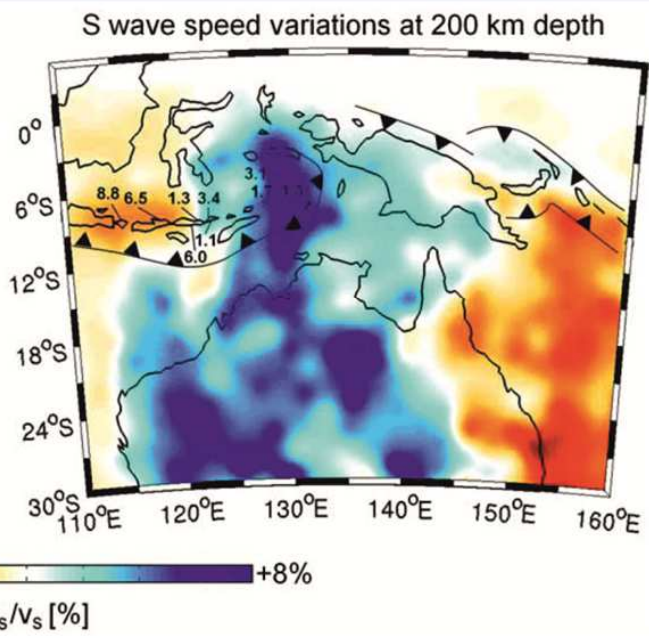

